\title{
SIMULATION-BASED ENVIRONMENTAL COST ANALYSIS FOR WORK-IN-PROCESS
}

\author{
Zhao, R. \\ Nagoya University, Graduate School of Economics and Business Administration, \\ Furo-cho, Chikusa-ku, Nagoya, Aichi, 464-8601, Japan \\ E-Mail: zhao.run@d.mbox.nagoya-u.ac.jp
}

\begin{abstract}
Under the modern manufacturing environment-oriented, both productivity and green environmental consideration are made in diverse phases of production operations. In this paper, in order to dynamically analyse and control changes of Work-In-Process (WIP) inventory level, a centralized fuzzy control method is proposed. Additionally, a new environmental management accounting method, Material Flow Cost Accounting (MFCA), is adopted to find and calculate environmental waste hiding in the production processes. Moreover, a simulation model integrated with these methods above is constructed. Based on the simulation data, sensitivity analysis between control factors of WIP inventory level and negative product costs ratio is made. To achieve a reasonable productivity as well as green environmental performance for this case, a corresponding optimized solving measure is put forward by means of OptQuest in the Arena simulation system. Through studying, the proposed control approach of WIP can reduce the green environmental cost, and improve production capacity.

(Received in February 2012, accepted in August 2012. This paper was with the author 2 months for 1 revision.)
\end{abstract}

Key Words: WIP, Material Flow Cost Accounting, Fuzzy Control, Simulation

\section{INTRODUCTION}

In order to satisfy the diversified demands of consumers and rapid response to market needs, multi-variety and small batch production system has been applied widely to obtain a great economic effect, and it can also significantly improve the capacity of production [1]. In this production system, work-in-process (WIP is called for short) buffers are inevitably set among workstations to balance production rhythm and guarantee productive stabilization by avoiding "block"/"starvation" in the production caused by machine breakdowns or defective production [2]. However, the current production practice shows that, owing to the differences in the processing sequence of part families, this production mode produces some difficulties in control of WIP: (1) Complex management of WIP buffers; (2) Inaccurate control of WIP level; (3) Occupation of much liquid capital. And, effective control of WIP inventory level is directly related to whether production capacity is improved or not.

Additionally, under the modern manufacturing environment, both green environmental consideration and productivity are made in diverse phases of production operations [3]. Achieving zero emission from production activities is forming a common view around the world. However, in the current manufacturing mode, because of the complex nature and randomicity of WIP control, high WIP inventory level can not only affect productivity, but also cause environmental cost as a result of inaccurate WIP control level, especially negative product cost such as raw material waste cost, energy waste cost, and idle processing cost and so on. Therefore, that how to control the WIP inventory level to improve the production capacity as well as to achieve green manufacturing effects is the important issue to be solved urgently in production research field. 
During production process, dynamic and stochastic changes of WIP inventory level complicate the production management. In the recent decade, many scholars have done a lot of researches on the control method and optimum capacity design of WIP inventory level. Diamantidis and Papadopoulos adopted Markov chain model to do an effective analysis on WIP buffer capacity [4]. Yang et al. presented a simulation optimization approach to resolve a constant work-in-process (CONWIP) strategy problem [5]. Lin et al. developed an algorithm integrating an artificial neural network and the sequential quadratic programming method to find an optimal WIP level [6]. Moreover, from the cost aspect, Betrand and Ooijen investigated work order release from an economic perspective to minimize the cost associated with the length of the customer order lead-time, the order tardiness and the work-in-process in the workshop [7]. However, these researches are mainly focused on optimization for WIP inventory level and production cost. As for the aspect of sensitivity to production capacity by analysing the dynamic or stochastic change of WIP inventory level, the research is rare.

Additionally, environment-oriented research of production process is a new trend. In order to achieve environmental sustainability, a new measure called green production has to be proposed. Ibrahim et al. have analysed and assessed some potential green sources, renewable sources and energy for products and production purposes [8]. Reibenschuh et al. and Pusavec and Kopac used advanced machining technologies to reduce production costs for creating less wastes $[9,10]$. Ahmed presented a system model for the new green manufacturing paradigm that it captures various planning activities to migrate from a less green into a greener and more eco-efficient manufacturing [11]. However, these researches mainly focused on green resources, green energy, green products and studied a macroscopic structure for entire green production process. They seldom attach importance to environmental effect by studying dynamic or stochastic change of production process in details, and also rarely emphasize the control of WIP inventory level which can cause environmental cost.

In this paper, therefore, a discrete simulation model for a certain pre-treatment workshop is developed, and the real-time change of WIP inventory level is dynamically tracked by monitoring the model running. In contrast to the traditional production research approaches, a centralized fuzzy control methodology is applied to manage WIP inventory level and its environmental cost is calculated by using a new environmental management accounting method called Material flow cost accounting (Called MFCA for short). In addition, the sensitivity of WIP to the green environmental performance is also analysed. From the standpoint of MFCA, a change law of negative product costs ratio with the changing of WIP inventory level is indicated. Furthermore, an optimized control approach of WIP inventory level is proposed to achieve both green environmental consideration and production capacity.

\section{BASIC DESCRIPTION OF THE CASE STUDY}

Group-Production Cell (Called GPC for short) is a specialized cellular production organization. It uses group technology to process part families with multi-variety and small batch in an appropriate region of workshop with corresponding manufacturing facilities performing similar processes [12]. In this production mode, in order to meet the processing demands for parts diversification, some WIP buffers are used to balance machining capability. So, the management and control for WIP in GPC is an important issue.

In this paper, a certain GPC in CD Shipyard's pretreatment workshop is studied for the case. In this GPC, it mainly comprises eight workstations that occupy a partial area of this workshop. Each independent processing in a workstation is handled by three machines or workers. According to the characteristics of part family, each workstation completes the processing task of various parts in a corresponding part family either in whole or in part. The processing time of each part on each workstation is different. In this case, three main part 
families $\left(F_{1}, F_{2}\right.$, and $\left.F_{3}\right)$ are chosen. Each part family is processed by different and independent production line. In the last workstation, each different part from these three part families is matched to be a set of component. Thinking of GPC's characteristics and ensuring its current logical structure, a simplified layout model for this GPC is shown in Fig.1.

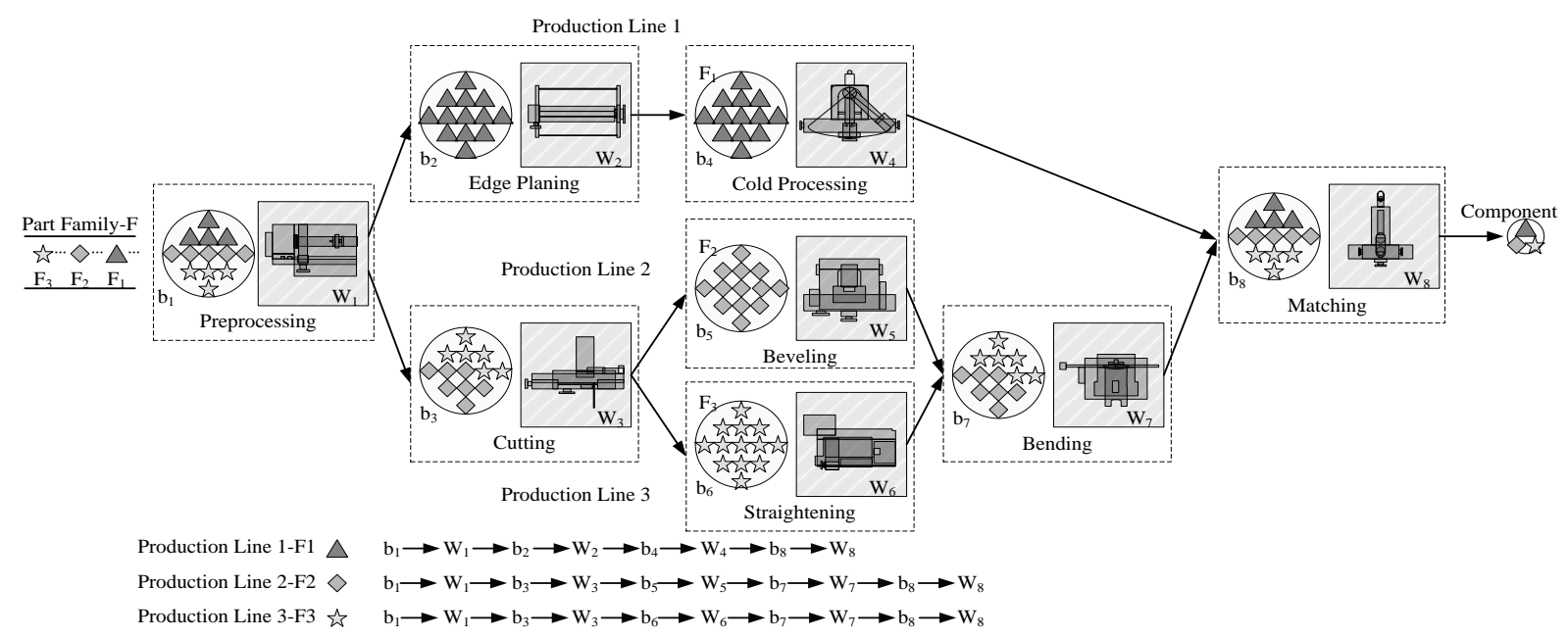

Figure 1: A simplified layout model for GPC.

From Fig.2, according to the actual production data, the Takt time of each workstation for the same production line is unbalanced and processing load is not consistent. These fluctuations of Takt time are mainly caused by different parts structures, the processing compatibility of machines for different parts and processing stability of machine. In order to reduce these fluctuations, a certain amount of WIP buffers are set in the upstream of the stations. However, though this setting improves the production stationarity, accurate control of WIP is still a serious problem. For instance, firstly, the current inventory level of WIP is very high. It still exits some cases that some upstream workstation is stopped optionally to reduce parts' input for downstream WIP. Secondly, too many parts in WIP buffers consume plenty of material cost, idle processing cost, and maintenance cost. Thirdly, processing these parts need lots of unnecessary electric energy and also cause raw material waste or auxiliary detergents waste. So, the production capacity and environmental cost need to be improved urgently.

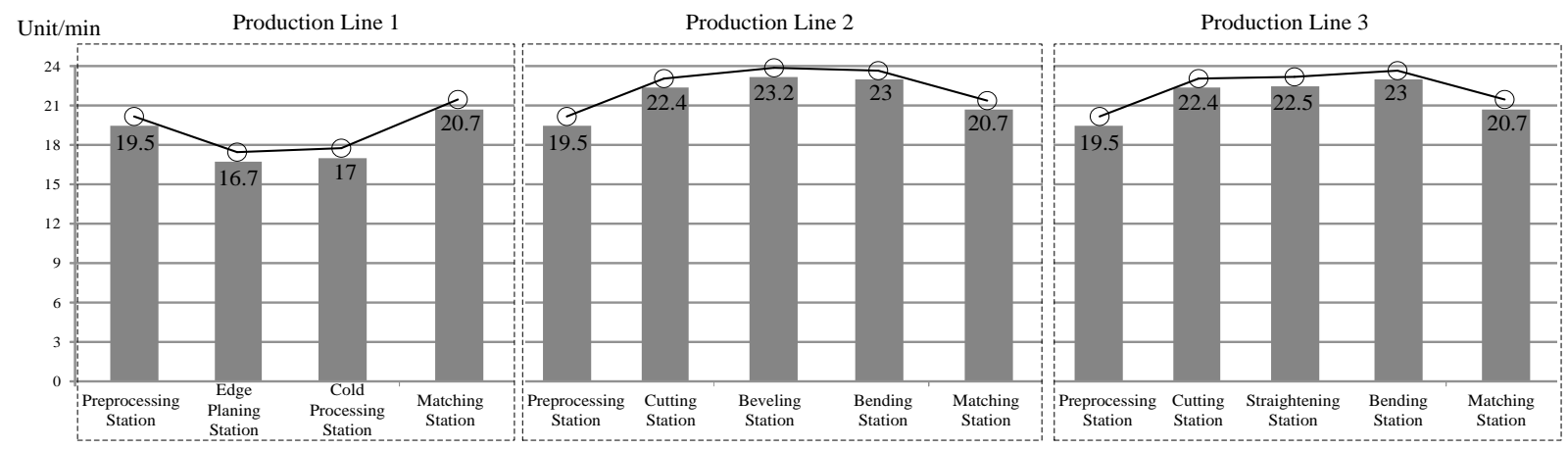

Figure 2: The Takt time of each workstation.

\section{APPROACH ANALYSIS}

In this paper, research object is a representative discrete production system, and its specific characteristics are illustrated in the Section 2. In order to resolve the current problems, firstly, a 
centralized fuzzy control methodology is used to simulate actual control manners for WIP by operators on site. It is also applied to adjust production ratio for workstation by examining inventory level of upstream WIP. The processing rhythm of production lines can be balanced and stability can be ensured. Secondly, MFCA is used to calculate some main production costs, specially, including unnoticed environmental costs that are hided under the production processes. Thirdly, a simulation model integrated with two approaches above is developed. And then, the simulation data is used to analyse sensitivity of WIP to environmental effect. Fourthly, an optimal solution is proposed. And a comparison is made to verify whether the production capacity and environmental cost is improved or not.

\subsection{A centralized fuzzy control methodology}

In the actual production workshop, operators on site cannot control the WIP inventory level in the form of an accurate quantity, but randomly adjust the production capacity in the manner of a random distribution by examining the inventory level of WIP buffer. This random distribution makes the production process more stochastic and also make system model more complex, but can describe the real situation clearly. Furthermore, a production system is usually viewed as a network of workstations and WIP buffers. The differences of items' structure in the part family make the processing time of machine be random, and also lead volatility disturbing the production stationarity. For the operators in the workstation, the easy way to balance the production line is to control WIP inventory level by examining the phenomena such as starvation/blocking that may occur in the upstream WIP buffer. On the other hand, in practice, a centralized control for WIP can be easily applied to manage WIP inventory level and also can avoid adverse impact caused by operators' separated adjustment. Therefore, in this paper, in view of actual conditions, a centralized control method of WIP inventory level is only taken account into and it is embedded into the computer simulation to research the production system. However, actually, stochastic of WIP control make it more difficult to simulate and control actual production.

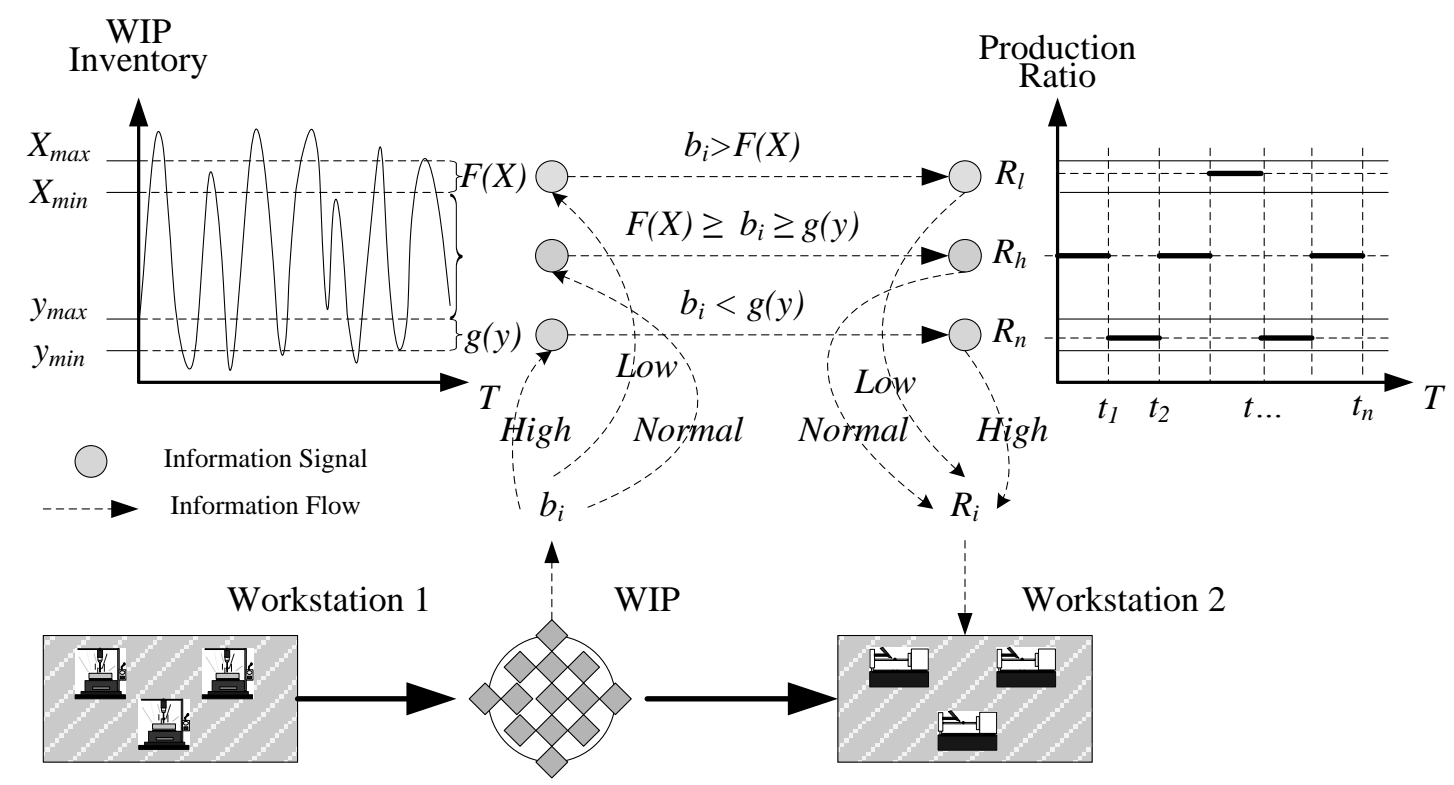

Figure 3: A fuzzy controller for WIP inventory level.

Fortunately, a fuzzy control methodology can easily resolve this problem, and keep the WIP inventory at a reasonable level [13]. So, in this paper, a fuzzy controller is set to simulate real control. In Fig. 3, wave shape curve shows the change of WIP inventory level with time. 
$F(X)$ and $g(y)$ are stochastic distribution functions of WIP control, and denote the highest and lowest threshold value of WIP inventory level. $X_{\min }, X_{\max }, y_{\min }, y_{\max }$ respectively obey these functions. If deviating from these threshold values that are assumed to be a certain distribution of random, the production ratio of downstream workstation will be adjusted to balance the production line. And these random distributions in the simulation model present a fuzzy control methodology, and this control is centralized. In fuzzy controller, the control policy is described by linguistic IF-THEN rules with appropriate mathematical meaning [14].The rule base of the control model contains rules of the following form:

$$
\begin{gathered}
\text { IF } b_{i} \text { is } B L^{(n)} \\
\text { THEN } r_{i} \text { is } P R^{(n)},
\end{gathered}
$$

where $n$ is the rule number $(n=1,2,3), i$ is the number of workstation, $B L$ is a linguistic value of the variable of WIP inventory level $b$ (upstream WIP of the workstation) with term set $B=\{$ Low, Normal, High $\}$. The production speed $r$ takes linguistic values of $P R$ from the term set $R=\{$ Low, Normal, High $\}$. The actual rulebase is presented in Table I.

Table I: Linguistic IF-THEN rules for fuzzy controller.

\begin{tabular}{|c|c|c|c|c|}
\hline RULE & \multicolumn{2}{|c|}{ IF $B L_{i}$} & \multicolumn{2}{|c|}{ THEN $R_{i}$} \\
\hline 1 & $b_{i}<g(y)$ & Low & $R_{l}$ & Low \\
\hline 2 & $F(X) \geq b_{i} \geq g(y)$ & Normal & $R_{n}$ & Normal \\
\hline 3 & $b_{i}>F(X)$ & High & $R_{h}$ & High \\
\hline
\end{tabular}

This centralized fuzzy control methodology can not only simulate the actual stochastic control for WIP by operators on site, but also can adjust production ratio by examining inventory level of upstream WIP.

\subsection{MFCA}

The control of WIP inventory level inevitably produces an influence on the green manufacturing. Environmental consideration and cost is realized gradually. MFCA is one of the environmental management accounting methods aimed to reduce environmental cost at the same time, as a tool of decision making by on-site manager. MFCA seeks to reduce cost through waste reduction or achieving zero emission and is adopted to realize the "harmony of environmental aspects and profitability", thereby improving productivity [3] [15].

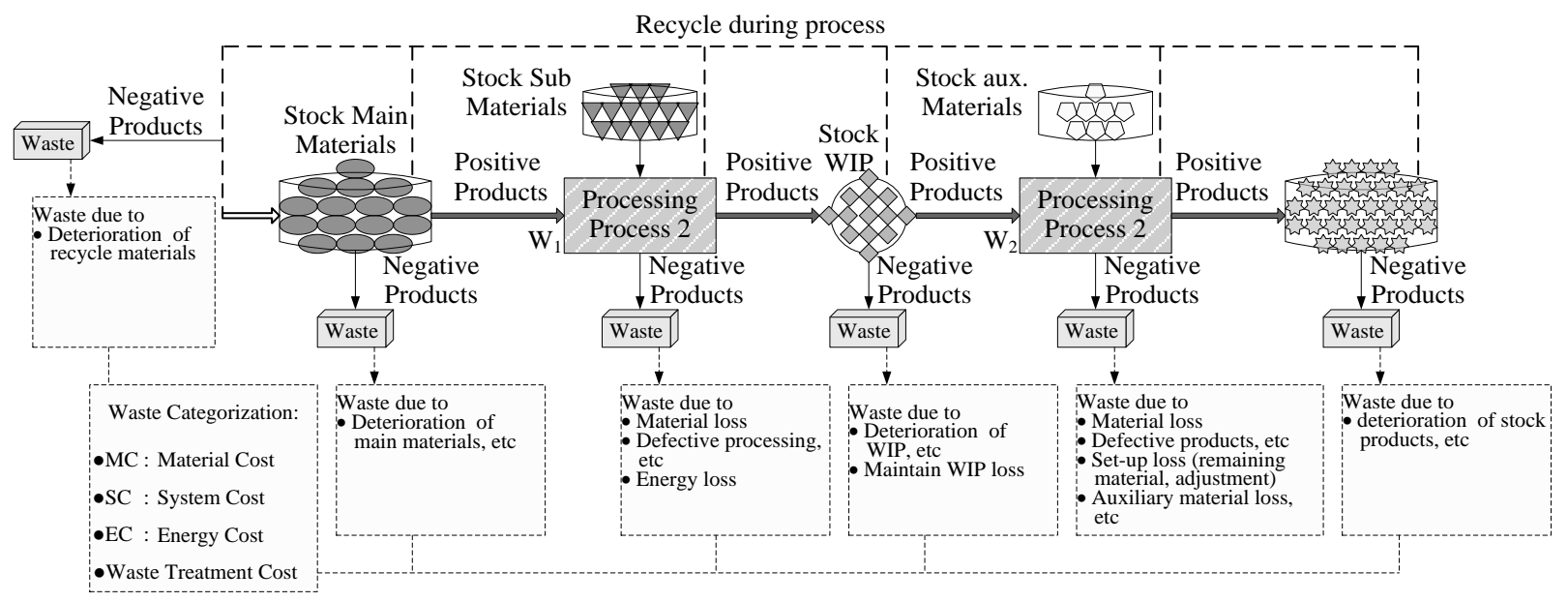

Figure 4: MFCA calculation flow and process. 
MFCA traces equally the both flows of final products and emissions (wastes) in processes. And, MFCA recognizes even the emission as one product. It calls products "positive products" and the emissions "negative products". Fig. 4 shows MFCA calculation flow and process. In this paper, the author adds the MFCA method into the simulation system to dynamically calculate environmental costs throughout all the processes with the change of WIP inventory level. According to rules of MFCA, the product cost is divided into positive and negative cost for calculation in terms of monetary unit. Comparing with the other account methods, MFCA makes negative products and some loss visible for each process. This visibility testifies that the change of WIP inventory level can not only make an influence on productivity, but also environmental cost.

\subsection{Simulation}

Aiming to resolve current problems in GPC, the centralized fuzzy control methodology is used to control change of WIP inventory level, and MFCA is adopted to calculate environmental cost. In this paper, one WIP managers group is set up to exam the WIP level in the manner of centralized management at regular intervals. The criterion of WIP inventory capacity is assumed to be a certain random distribution $F(X)$ and $g(y)$. After finding some WIP inventory level is high than $F(X)$ or lower than $g(y)$, the downstream workstation's production ratio will be adjusted. During the processing, MFCA is used to calculate positive and negative products cost. The simulation logic is shown in Fig. 5.

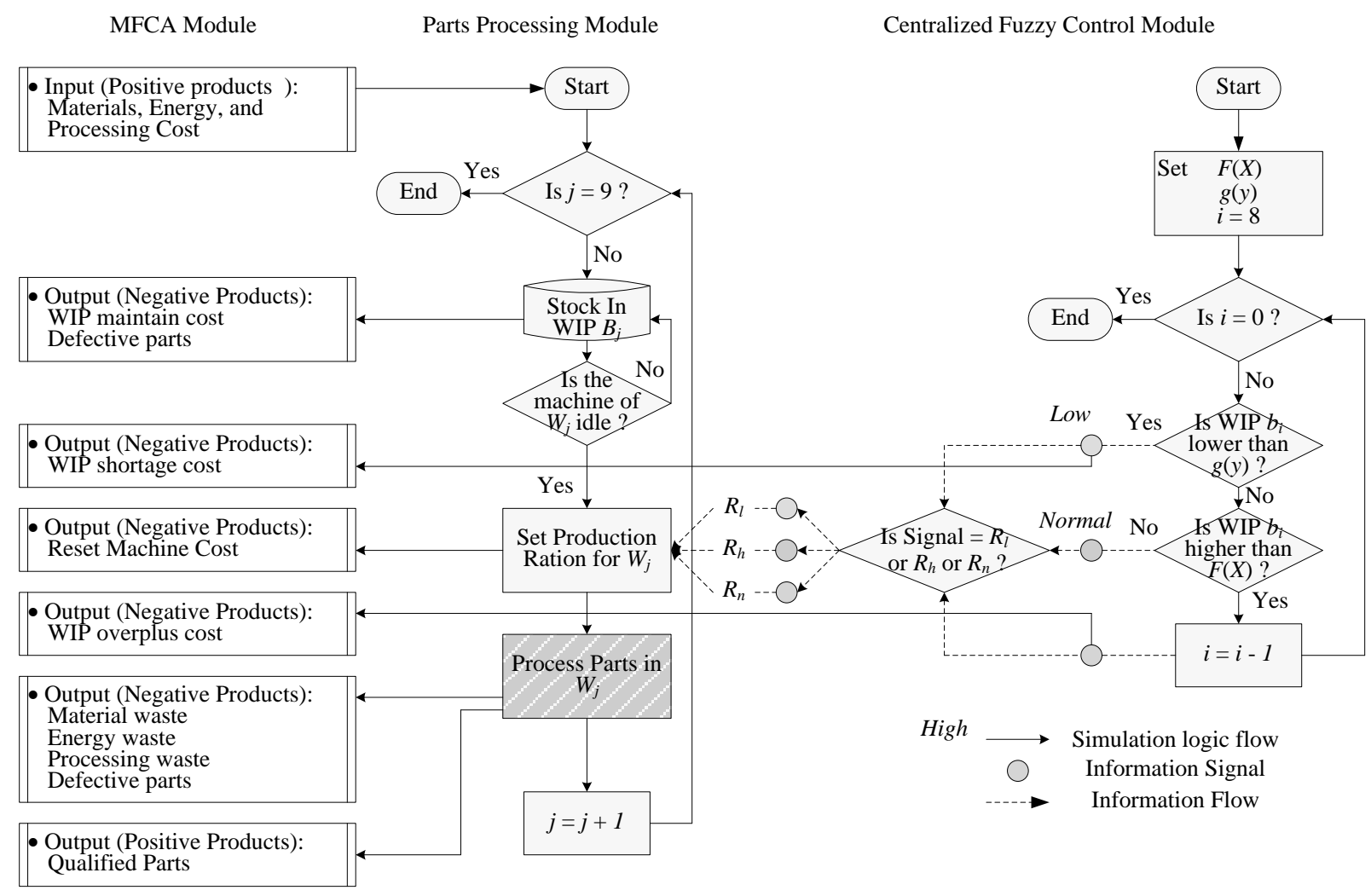

Figure 5: The simulation logic.

As presented in Fig.5, system simulation model is composed by fuzzy control logic module MFCA module, and Parts processing module. Fuzzy Control logic module monitor the change of WIP inventory level at a certain examining time in the manner of centralized control, and decide to adjust production speed. Parts processing module operate the 
production system. MFCA module calculates all the manufacturing costs including environmental cost.

After collations and sorting out, main data is embedded into the simulation model. All the parts in different part family enter this system at a random distribution. Each WIP buffer is set in upstream of the each workstation, and each a group of five parts in a WIP buffer is placed in a pallet. In order to avoid "block" or "starvation", a certain WIP inventory level is set. The lowest and highest level of fuzzy control both obeys uniform distribution, i.e. $F(X)=\mathrm{UNIF}$ $\left(X_{\min }, X_{\max }\right), g(y)=\mathrm{UNIF}\left(y_{\min }, y_{\max }\right)$. The difference between minimum and maximum value of the uniform distribution is 5 that are equal to the pallet capacity. According to the fuzzy control signal, the number of machines in a workstation is reset to adjust production speed. Processing time all obeys random distribution. Because of the relative heaviness of each steel part, it is transferred to downstream workstation by the fork truck.

In this paper, Arena simulation platform is used to build simulation model, and simulation running is based on practical production data of a month. Each workday has 24 hours and CD shipyard uses three-shift workday system because of prosperous shipbuilding business at present in China. So, the simulation running-length is 43200 minutes. In order to avoid the impact of data deviation on simulation system performance due to initial status, steady-state simulation is appropriate and warm-up period is 5000 minutes. Additionally, in order to make simulation more stochastic and independent and to get a narrower $95 \%$ prediction interval, number of replication is set by 20 .

\subsection{Cost calculation}

One object of this paper is to show the sensitivity of negative cost to change of WIP inventory level. Due to the adjustment of production speed, positive products inputs inevitable change a lot. It is difficult to set a fixed criterion to evaluate negative cost in a fixed input. Thus, a negative cost ratio is adopted in this paper that the ratio is the value of negative cost divided by positive products input. And, the lower the ratio is, the better control efficiency of WIP inventory level is, and the higher production capacity is. To calculate this ratio, first step is to collect the positive products input, and then calculating negative products cost is the second step. All the cost is in form of monetary units.

\section{Step 1 - Calculate the Positive Products Cost}

Positive products input are composed by three parts: material cost, process cost, and energy cost.

where:

$$
T P C=M c+P c+E c
$$

TPC: total positive products input cost,

$M c$ : material cost,

$P c$ : process cost,

Ec: energy cost.

\section{Step 2 - Calculating Negative Products Cost}

Negative Products Cost are composed by eight parts by maintain cost of WIP inventory, cost of lower than safe WIP level $g(y)$, cost of higher than safe WIP level $F(X)$, cost of resetting machine set, cost of part weight waste, cost of part processing waste, cost of part energy waste, cost of defective part.

where:

$$
T N C=M I c+L M I c+H M I c+R M c+W W c+P W c+E W c+D P c
$$

TNC: total negative products cost.

MIc: maintain cost of WIP inventory, 
LMIc: cost of lower than safe WIP level $g(y)$,

HMIc: cost of higher than safe WIP level $F(X)$,

$R M c$ : cost of resetting machine set,

$W W c$ : cost of part weight waste,

$P W c$ : cost of part process waste,

$E W c$ : cost of part energy waste,

$D P c$ : cost of defective part.

Table II: Cost Categorization Based on MFCA.

\begin{tabular}{|c|c|c|c|c|}
\hline & & Input & Output & \\
\hline \multirow{3}{*}{ Positive Products Cost } & $\mathrm{MC}$ & $M c$ & / & \multirow{3}{*}{$T P C$} \\
\hline & $\mathrm{SC}$ & $P c$ & I & \\
\hline & $\mathrm{EC}$ & $E c$ & I & \\
\hline \multirow{3}{*}{ Negative Products Cost } & $\mathrm{MC}$ & $\overline{1}$ & $W W c$ & \multirow{3}{*}{$T N C$} \\
\hline & $\mathrm{SC}$ & / & Mic, LMIc, HMIc, RMc, PWc, DPc & \\
\hline & $\mathrm{EC}$ & / & $E W c$ & \\
\hline
\end{tabular}

These negative products cost can be calculated easily by simulation system. Based on step 1 and step 2 above, according to MFCA, all kinds of cost are shown in Table II.

\section{Step 3 - Calculating Negative Cost Ratio}

$$
R=\frac{T N C}{T P C}
$$

where:

$R$ : negative cost ratio.

According to the centralized fuzzy control method and simulation requirement about time, $R$ can be seen as a function of the variables $t, y$ and $X$. So, $R$ is denoted as $R(t, g(y), F(X))$. The change of WIP inventory level is related to these variables closely.

\section{DATA ANALYSIS}

\subsection{Sensitivity analysis}

Some factors such as the differences in worker qualification and practical technique can affect the production performances. But, in this paper, in order to study the efficiency of fuzzy control method, only three related variables, $t$ (examination time of WIP inventory level), $g(y)$ (the lowest fuzzy control threshold value of WIP), and $F(X)$ (the highest fuzzy control threshold value of WIP) are considered. Alteration of these variables can change the $R$. It also can be seen that $R$ is not a continuous derivative function, and its partial differential equation of first order for $t, g(y)$ or $F(X)$ cannot be gotten. So, sensitivity analysis can show the impact degree of random variable $t, g(y)$ and $F(X)$. The other factors are not considered.

$$
\begin{gathered}
\left|\Delta R_{(\Delta t)}\right|=|R(t)-R(t \pm \Delta t)| \\
\left|\Delta R_{(\Delta y)}\right|=|R[g(y)]-R[g(y \pm \Delta y)]| \\
\left|\Delta R_{(\Delta X)}\right|=|R[F(X)]-R[F(X \pm \Delta X)]|
\end{gathered}
$$


$\Delta R_{(\Delta t)}, \Delta R_{(\Delta y)}, \Delta R_{(\Delta X)}$ denote that changing value of $R$ with the minimal change of $t, g(y)$ or $F(X)$ and the other two fixed variables. If $\Delta R$ is more obvious relatively, it indicates that sensitivity degree is high, but low. For an example, starting with the lowest $y_{\min }, y$ increases progressively to the highest $y_{\max }$ by a small fixed increment $\Delta y$. In the interval $\left(y_{\min }, y_{\max }\right)$, each $\Delta R_{(\Delta y)}$ is calculated with each $\Delta y$ increase. Among these $\Delta R_{(\Delta y)}$, we can get a lowest $R$ and can also the highest sensitive degree in a certain $y$. If the increment change of these $\Delta R_{(\Delta y)}$ is so little, it can be seen that highest sensitive is not obvious, i.e. the control of $g(y)$ cannot improve $R$ effectively. The other two variables $t$ and $\mathrm{F}(X)$ is the same as $g(y)$.

In this study, the change of WIP is mainly affected by three factors. The sensitivity analysis regarding with these factors is made based on the simulation data. In order to accurately analyse the sensitivity, one factor is studied and the others must keep steady.

From simulation data, it can be seen that average WIP level is about 50, and $t$ should start with the $3^{\text {rd }}$ hour. And, in the practice, the lowest threshold value of $g(y)$ must be 5 at least, which is equal to the quantity of a pallet. So, the sensitivity analysis about examining time $t$ must keep the other two factors the $g(y)=\operatorname{UNIF}(5,10)$, and $F(X)=\operatorname{UNIF}(50,55)$ be steady.

In Fig. 6, the lower line shows the $R$ change of each $t$ point which changes from the $4^{\text {th }}$ hour to $30^{\text {th }}$ hour, and each $R$ is the value which subtracts the $t=3^{\text {rd }}$ value. The upper line shows the change of $\Delta R{ }_{(\Delta t)}$ based on each fine increment $\Delta t(\Delta t=1$ hour). Seeing the upper line, the change of $\Delta R_{(\Delta t)}$ is uneven. Before $t=8, \Delta R_{(\Delta t)}<0$, it denotes that $R$ gradually becomes lower and amplitude of variation is large. It means that sensitivity degree is high before $t=8$. After $t=$ $8, \Delta R>0$ mostly, it denotes that $R$ gradually becomes larger, and the zigzag change means sensitivity degree is not high. When $t=8, \Delta R=0$, it means the $R$ is at the lowest value. These change is also can be testified from lower line. So, it shows that control of $t$ should be close to 8 , and the change speed of $R$ before $t=8$ is larger than after $t=8$ in the same fine increment $\Delta t$.

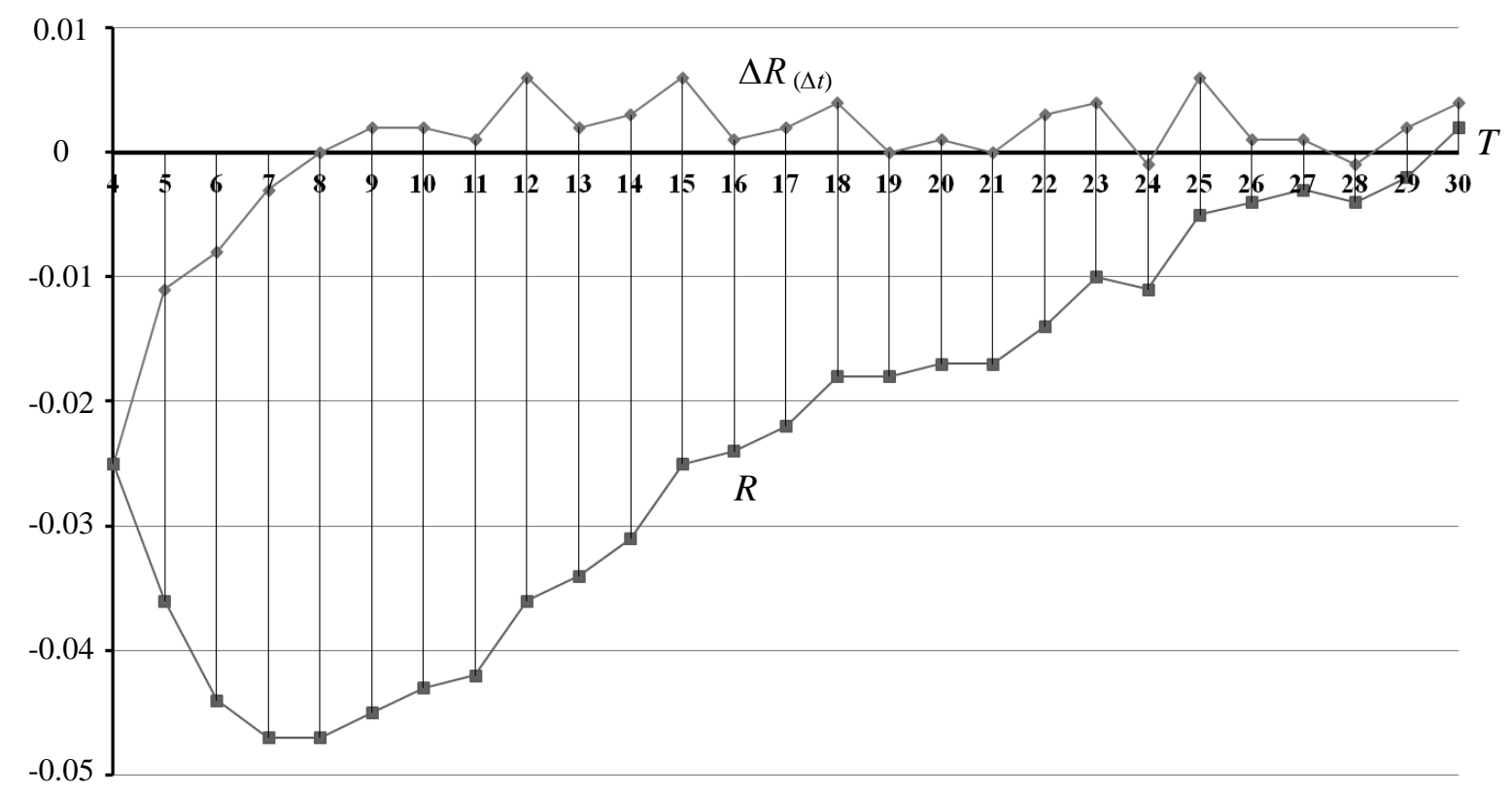

Figure 6: Sensitivity analysis about examining time $t$.

For the sensitivity analysis about $g(y)$ in Fig. 7, we keep $t=8, \Delta y=5$ and study the changes from $F(X)=\operatorname{UNIF}(115,120)$ to UNIF $(50,55)$. Seeing Fig. 8 , all the curves show that with the decrease of $g(y)$ value, the change of curve became gently. And based on the same $F(X), \Delta R_{(\Delta y)}$ of the same $g(y)$ point became larger with increase of $g(\mathrm{y})$ and the lowest $R$ occurs in curve $g(y)$ $=\operatorname{UNIF}(5,10)$. It shows that sensitivity degree became larger with increase of $g(\mathrm{y})$ value. So, it means that we should keep the $g(\mathrm{y})$ lower. 


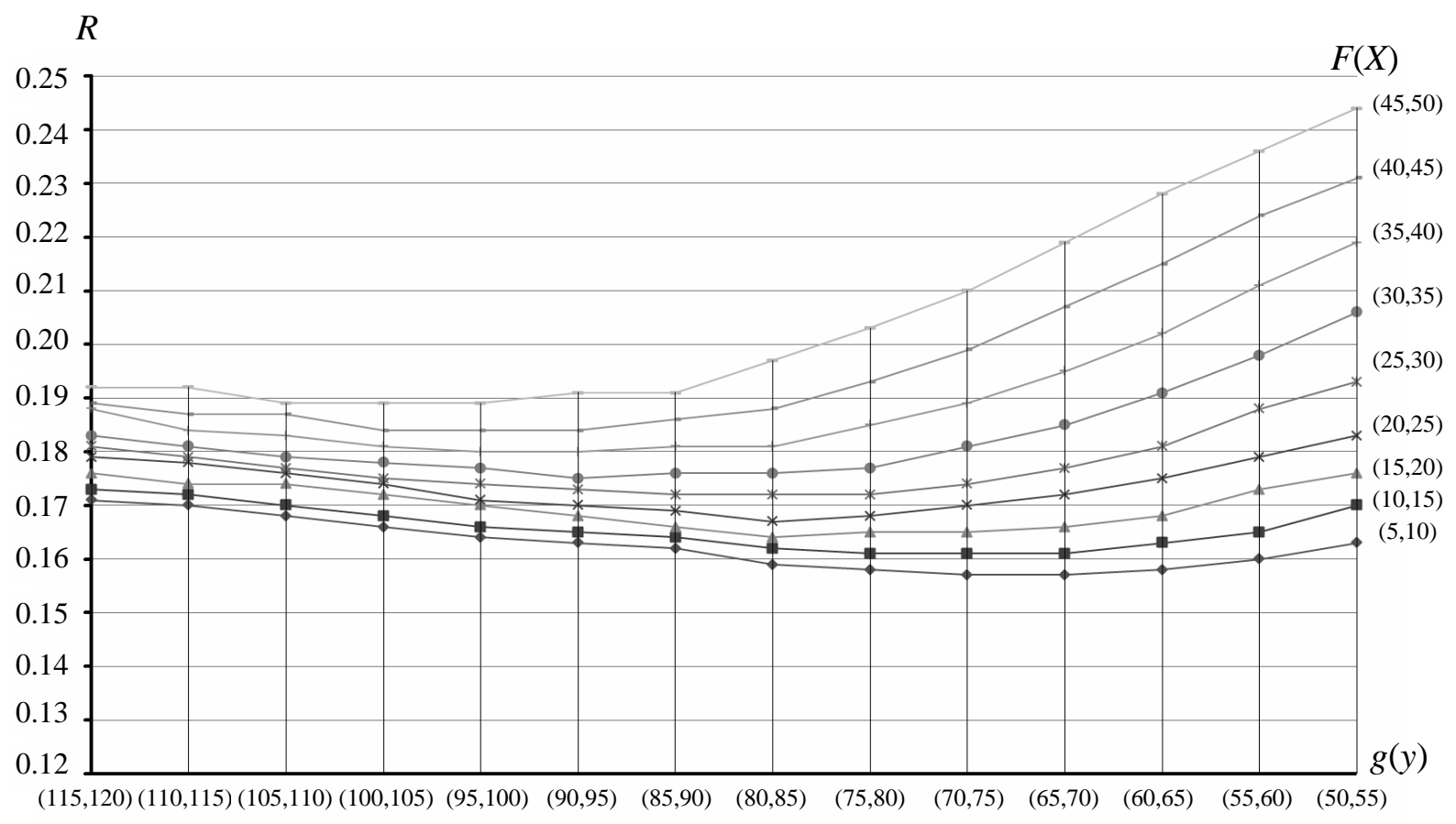

Figure 7: Sensitivity analysis about $g(y)$.

Fig. 8 shows the sensitivity analysis about $F(X)$, keeping the $t=8, \Delta X=5$ and study the changes from $g(y)=\operatorname{UNIF}(5,10)$ to $\operatorname{UNIF}(55,60)$. It is can be seen that the lowest point of all the curves correspond to $g(y)=\operatorname{UNIF}(5,10)$. With the increase of $g(y)$, the lower $F(X)$, the higher $R$ is. It also shows that sensitivity degree became larger with decrease of $F(X)$.

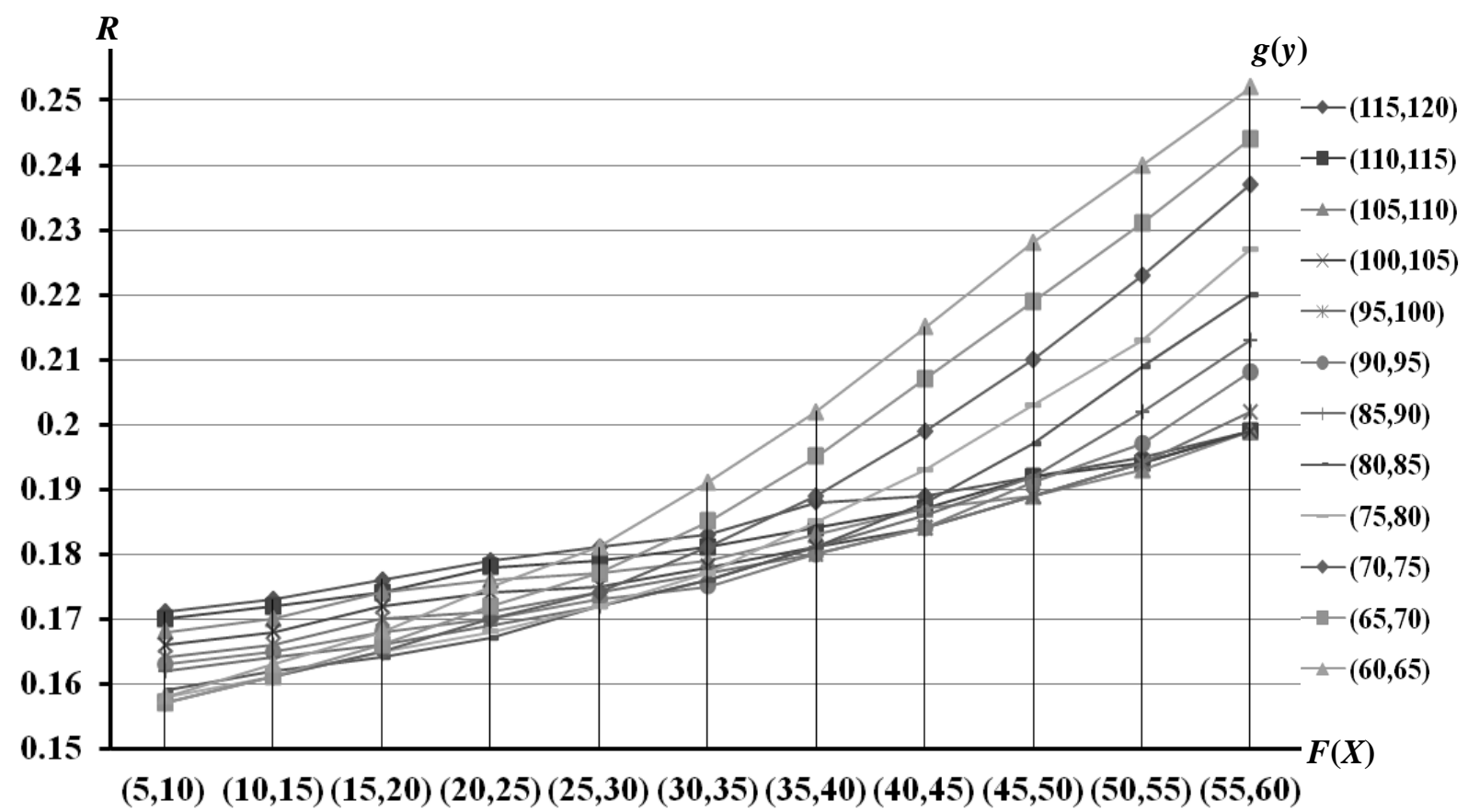

Figure 8: Sensitivity analysis about $F(X)$.

By analysing sensitivity, we can clearly see that the how these factors affect the $R$, and also can know the effect degree. And, in the practice, the managers can control $R$ with changing these factors, master which factor can bring about larger changes, and predict the change trend. 
At the same time, the workers can reasonably adjust the WIP inventory level of upstream to improve $R$ by resetting production speed, and know the change amplitude of $R$ by adjusting the WIP. Additionally, analysing sensitivity can provide an effective and fast search route for optimization analysis.

\subsection{Sensitivity analysis about optimization}

Optimization analysis can get an optimal $t, g(\mathrm{y})$ and $F(\mathrm{X})$ to achieve the lowest $R$. The manager and worker can use this optimal solution to manage WIP control as a base. OptQuest is a software package embedded in the Arena. It applies Tabu Search and Scatter Search to get the optimal solution. In this paper, the author uses this OptQuest to find the optimal control of WIP with considering change law of sensitivity analysis about three factors, which can limit down the search scope. And, according to the expressions in section 3.4., optimal $R$ and constraint conditions can be presented in OptQuest as follows:

$$
\begin{array}{ll} 
& \min R=\min \frac{T N C}{T P C} \\
& y_{\text {max }}=y_{\text {min }}+5 \\
\text { s.t. } & X_{\text {max }}=X_{\text {min }}+5 \\
& y_{\text {max }}<X_{\text {min }} \\
& t=3,4,5 \cdots, 30
\end{array}
$$

After searching, an optimal solution is achieved: $t=7$ hour, $g(y)=\mathrm{UNIF}(5,10), F(X)=$ $\operatorname{UNIF}(55,60)$, and then $R=0.158$. In the same way, the sensitivity analysis about optimal solution can also be gotten, as seen in Fig. 9.

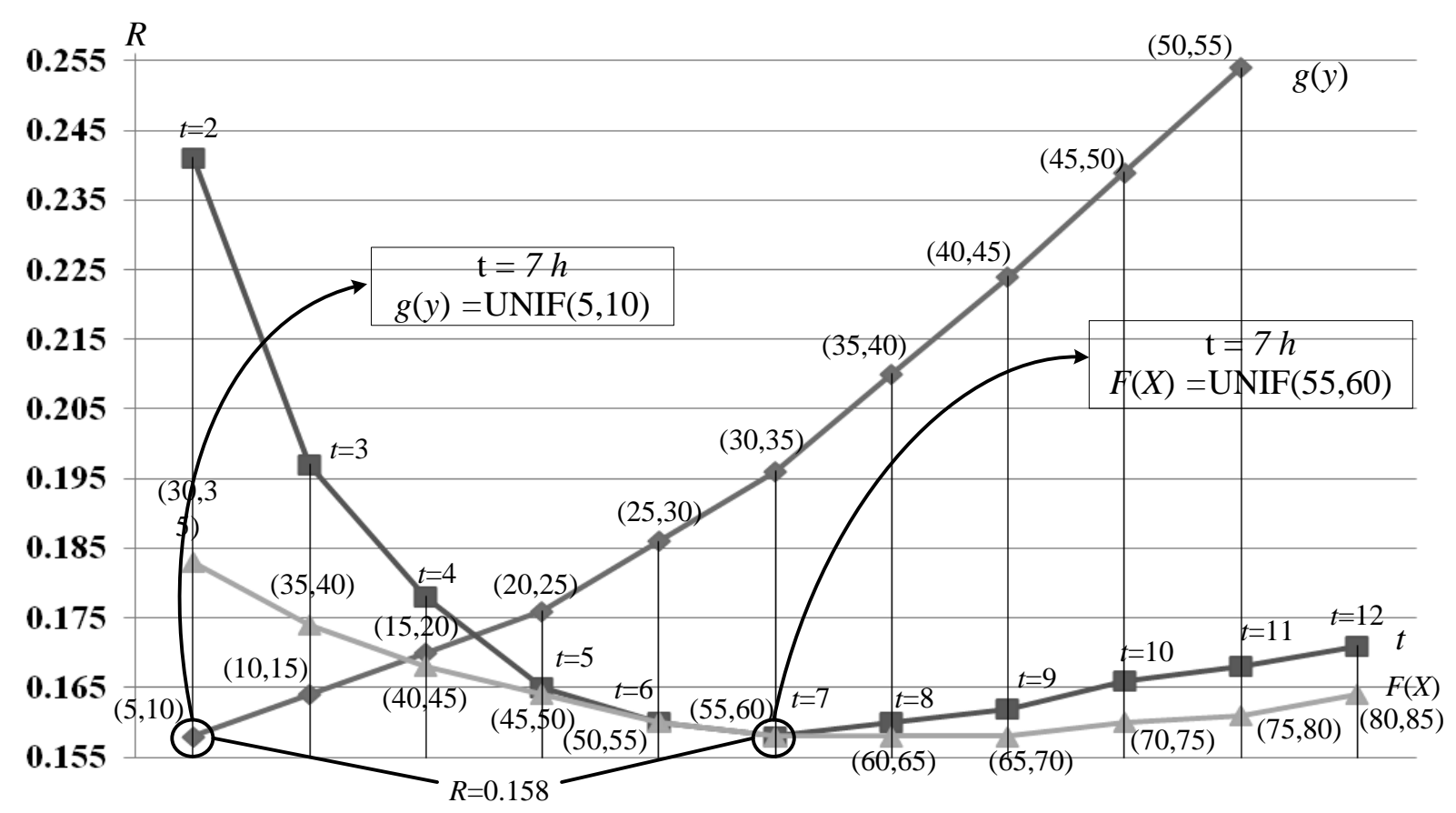

Figure 9: Sensitivity analysis about optimal solution.

The point drawn by circle is the optimal value $R=0.158$ about three curves. The ' $\mathbf{n}$ ' curve denotes the sensitivity analysis about $t$, and shows a sag curve. The ' $\mathbf{\Lambda}$ ' curve denotes the sensitivity analysis about $g(y)$, and also shows a ascend curve. The ' curve denotes the sensitivity analysis about $F(X)$, and shows a sag curve. For these curves, the optimal value is 
the lowest point, and the ' $\mathbf{\Delta}$ ' curve is most flat, i.e. the sensitivity about $F(X)$ is lowest. Additionally, the curvation of ' curve is the biggest, i.e. the sensitivity about $g(y)$ is the highest. So, from Fig. 9, we can consider that the control about $g(y)$ is more effective than other two factors. And, for the managers or workers, in the same situation, the control priority about these three factors is: firstly, $g(y)$; secondly, $t$; finally, $F(X)$.

\subsection{Comparison}

After sensitivity and optimization analysis, comparison with original model (AS-IS), model applying MFCA (TO-BE) and optimal model, these three models can be gotten. By this comparison, how the production capacity and environmental performance can be improved, can be seen clearly in Table III.

Table III: Comparison based on output data

\begin{tabular}{|c|c|c|c|c|}
\hline & & AS-IS Model & TO-BE Model & Optimal Model \\
\hline \multirow{4}{*}{ Positive Products Cost } & MC & 3304215.65 & 3267720.31 & 3567972.28 \\
\cline { 2 - 5 } & SC & 265691.12 & 240773.04 & 400084.87 \\
\cline { 2 - 5 } & EC & 137953.71 & 129730.18 & 140409.32 \\
\cline { 2 - 5 } & Total & 3707860.48 & 3638223.53 & 4108466.47 \\
\hline \multirow{4}{*}{ Negative Products Cost } & MC & 432109.83 & 468605.17 & 505864.96 \\
\cline { 2 - 5 } & SC & 342976.47 & 367894.55 & 256389.12 \\
\cline { 2 - 5 } & EC & $/$ & 8223.53 & 9071.32 \\
\cline { 2 - 5 } & Total & 775086.3 & 844723.25 & 771325.4 \\
\hline Negative Cost Ratio & $R$ & 0.1729 & 0.1884 & 0.158 \\
\hline Production Capacity & $C$ & 10666 & 10666 & 11968 \\
\hline
\end{tabular}

From Table III, traditional accounting method about AS-IS model show that $R=0.1729$, and the production capacity of positive products number is 10666. After using MFCA, it shows that $R$ is changed to 0.1884 . So MFCA are easier to find problem that many negative products cost is hided, and the amount of difference in cost is up to $¥ 70,000$. After improvement adopting MFCA and fuzzy centralized control methodology, $R$ is down to 0.158 , and capacity is up to 11968 . Comparing with TO-BE model, it can be seen that, in the same work time, cost of positive products increases $12.93 \%$, capacity increases $12.21 \%$, but cost of negative products decrease $8.69 \%$. So, the effect of improvement is very obvious.

\section{CONCLUSIONS}

In this paper, the centralized fuzzy control methodology is used to control the change of WIP inventory level, and MFCA is adopted to calculate system environmental cost by adjusting production capacity. According to comparison results in section 4.3, it is easily seen that the centralized fuzzy control methodology can exactly simulate WIP control by on-site operators. Also, it can adjust production ratio according to the WIP inventory level to balance production line and increase production capacity. For the MFCA, this method can be used to calculate environmental cost hiding in the production processes by the simulation for each 
process. Additionally, through sensitivity analysis with regard to three factors, $t, g(y)$ and $F(X)$, it can be found that the fine increment or decrement change of each factor can lead a relative big change of $R$. And, the sensitivity analysis can also make a fast search route to get the optimal solution by OptQuest software package. These change laws of sensitivity analysis as well as the optimal solution give the managers and worker on-site an easy and effective control method of WIP inventory level to achieve a good performance considering production capacity and environmental cost. Comparing with three models (AS-IS model, TO-BE model and optimal model), a conclusion is made that the methods for controlling WIP applied in this paper really improve the production capacity as well as reduce green manufacturing cost that it is a scientific issue proposed in section 1.

\section{ACKNOWLEDGMENTS}

This research was supported by Grant-in-Aid for Asian CORE Program "Manufacturing and Environmental Management in East Asia" of Japan Society for the Promotion of Science (JSPS).

\section{REFERENCES}

[1] Gong, B. Z. (2002). The processing of parts with group technology in an individual CNC machining center, Journal of Material Processing Technology, Vol. 129, No. 1-3, 645-648, doi:10.1016/S0924-0136(02)00672-6

[2] Luca, B. (2011). Determining the optimal dimension of a work-in-process storage area, International Journal of Production Economics, Vol. 131, No. 2, 483-489, doi:10.1016/j.ijpe.2011.01.014

[3] Ministry of the Environment, Government of Japan (2009). MFCA guideline

[4] Diamantidis, A. C.; Papadopoulos, C. T. (2006). Markovian Analysis of A Discrete Material Manufacturing System with Merge Operations, Operation-dependent and Idleness Failures, Computers \& Industrial Engineering, Vol. 50, No. 4, 466-487, doi:10.1016/j.cie.2005.04.004

[5] Pettersen, J.-A.; Segerstedt, A. (2009). Restricted work-in-process: A study of differences between Kanban and CONWIP, International Journal of Production Economics, Vol. 118, No. 1, 199-207, doi:10.1016/j.ijpe.2008.08.043

[6] Lin, Y. H.; Shie, J. R.; Tsai, C. H. (2009). Using an artificial neural network prediction model to optimize work-in-process inventory level for wafer fabrication, Expert Systems with Applications, Vol. 36, No. 2, Part 2, 3421-3427, doi:10.1016/j.eswa.2008.02.009

[7] Betrand, J. W. M.; van Ooijen, H. P. G. (2008). Optimal work order release for make-to-order job shops with customer order lead-time costs, tardiness costs and work-in-process costs, International Journal of Production Economics, Vol. 116, No. 2, 233-241, doi:10.1016/j.ijpe.2008.08.055

[8] Gonzalez-Garcia, S.; Feijoo, G.; Heathcote, C.; Kandelbauer, A.; Moreira, M. T. (2011). Environmental assessment of green hardboard production coupled with a laccase activated system, Journal of Cleaner Production, Vol. 19, No. 5, 445-453, doi:10.1016/j.jclepro.2010.10.016

[9] Reibenschuh, M.; Cus, F.; Zuperl, U. (2011). Turning of high quality aluminium alloys with minimum costs, Technical Gazette, Vol. 18, No. 3, 363-368

[10] Pusavec, F.; Kopac, J. (2011). Sustainability Assessment: Cryogenic Machining of Inconel 718, Strojniski vestnik - Journal of Mechanical Engineering, Vol. 57, No. 9, 637-647, doi:10.5545/sv-jme.2010.249

[11] Deif, A. M. (2011). A system model for green manufacturing, Journal of Cleaner Production, Vol. 19, No. 14, 1553-1559, doi:10.1016/j.jclepro.2011.05.022

[12] Gajendra, K. A.; Divakar, R. (2000). The trade-off between intracell and intercell moves in group technology cell formation, Journal of Manufacturing Systems, Vol. 19, No. 5, 305-317 
[13] Tsourveloudis, N. C.; Dretoulakis, E.; Ioannidis, S. (2000). Fuzzy work-in-process inventory control of unreliable manufacturing systems, Information Sciences, Vol. 127, No. 1-2, 69-83, doi:10.1016/S0020-0255(00)00030-X

[14] Driankov, D.; Hellendoorn, H.; Reinfrank, M. (1993). An Introduction to Fuzzy Control, Springer-Verlag, Berlin

[15] Nakajima, M. (2009). Evolution of Material flow cost accounting (MFCA): Characteristics on Development of MFCA Companies and Significance of Relevance of MFCA, Kansai University Review of Business and Commerce, No. 11, 27-46 